\title{
Avaliação da teoria de ordem reduzida G3(MP2)//B3 com bases de Dunning em cálculos de afinidade protônica
}

\author{
Gerlane Bezerra da Silva ${ }^{1}$ (IC) ${ }^{*}$, Régis Casimiro Leal ${ }^{1,2}$ (PQ) \\ *e-mail: gerlanebezerra17@live.com \\ ${ }^{1}$ Instituto Federal de Educação, Ciência e Tecnologia do Rio Grande do Norte (IFRN), 59215-000, Nova \\ Cruz - RN, Brasil \\ ${ }^{2}$ Instituto de Química, Universidade Estadual de Campinas (UNICAMP), 13084-970, Campinas, São \\ Paulo - SP, Brasil \\ Palavras-chave: Afinidade protônica, bases de Dunning, teoria G3(MP2)//B3
}

\section{Introdução}

Combinações de diferentes níveis de teoria com conjuntos de funções de base de natureza variada vêm sendo utilizados, com frequência, principalmente na obtenção de propriedades termoquímicas com precisão elevada [1]. Essas combinações usam a adição de correções a uma energia eletrônica de referência e são conhecidos como métodos compostos, destacando-se as teorias Gaussian-n (Gn) e os métodos CBS [2].

Silva e Leal [3] mostraram que é viável combinar bases de Dunning ao invés da tradicional base 6-31G(d) de Pople, no método composto G3(MP2)//B3. Conseguiu-se uma redução em até $50 \%$ nos desvios, em cálculos de energia de ionização $\left(\mathrm{EI}_{0}\right)$ e afinidade eletrônica $\left(\mathrm{AE}_{0}\right)$ para átomos do conjunto teste $\mathrm{G} 3 / 05$, em relação às teorias G3(MP2)//B3 [4] e G3(MP2)//B3-CEP [5]. Portanto, surge agora a necessidade de verificar o comportamento na obtenção de propriedades em sistemas moleculares.

A afinidade protônica (A.P.) é considerada uma importante propriedade de interesse químico. Dentre as várias definições encontradas na literatura [6,7], a A.P. pode ser determinada simplesmente como a variação de entalpia padrão da reação de desprotonação de um sistema químico qualquer (X), conforme Equação 1.

$$
\mathrm{HX}_{(\mathrm{g})} \rightarrow \mathrm{H}^{+}{ }_{(\mathrm{g})}+\mathrm{X}_{(\mathrm{g})}^{-} \quad \text { A.P. }=\Delta_{\mathrm{r}} \mathrm{H}^{-}=\mathrm{H}_{\mathrm{X}^{(\mathrm{g})}}^{-}-\mathrm{H}_{\mathrm{HX}(\mathrm{g})}
$$

Nesse trabalho avaliou-se a combinação de bases de Dunning, $c c-p V n Z$ e aug$c c-p V n Z$, na teoria de ordem reduzida G3(MP2)//B3 para obtenção da A.P. do conjunto teste G3/05 [8] frente aos dados experimentais, bem como em relação ao desempenho das outras teorias equivalentes $[4,5]$.

\section{Detalhes Computacionais}

Todas as etapas para obtenção da energia G3(MP2)//B3 estão bem estabelecidas na referência [4]. Essa teoria utiliza dois conjuntos de bases, 6-31G(d) e G3MP2large. O conjunto de base 6-31G(d) é utilizado para obtenção da geometria (B3LYP), no cálculo das frequências vibracionais (B3LYP) e de correlação eletrônica (QCSID(T)). Neste trabalho substituiu-se o conjunto 6-31G(d) pelos conjuntos de base de correlação consistente desenvolvidos por Dunning et al., mantendo os demais procedimentos para obtenção da energia. Assim, os novos procedimentos aqui testados serão denominados G3(MP2)//B3-D, onde "D" faz referência aos conjuntos de base desenvolvidos por Dunning e colaboradores. Todos os cálculos foram realizados através do Gaussian 09w.

\section{Resultados e Discussões}

A Tabela 1 sumariza os valores de A.P. experimentais e calculados, o desvio médio (Desv.) e a estimativa do desvio padrão (Std.) são mostrados ao final da tabela. Os menores Desvs., em relação aos dados experimentais, são alcançados quando se usa o conjunto cc-pVQZ (ou 5z) superando de forma satisfatória as teorias correspondentes. 
Tabela 1. Afinidade protônica experimental e calculada (em kcal.mol ${ }^{-1}$ ) com os métodos compostos G3(MP2)//B3-CEP, G3(MP2)//B3 e G3(MP2)//B3-D

\begin{tabular}{|c|c|c|c|c|c|c|c|}
\hline \multirow{2}{*}{ Moléculas } & \multirow{2}{*}{ Expt. $^{[9]}$} & \multicolumn{4}{|c|}{ G3(MP2)//B3-D } & \multirow{2}{*}{$\begin{array}{c}\text { G3(MP2)// } \\
\text { B3 }\end{array}$} & \multirow{2}{*}{$\begin{array}{l}\text { G3(MP2)// } \\
\text { B3-CEP }\end{array}$} \\
\hline & & cc-pVDZ & cc-pVTZ & cc-pVQZ & cc-pV5Z & & \\
\hline $\mathrm{H}_{2} \mathrm{O}$ & 165,1 & 163,6 & 163,5 & 163,6 & 163,7 & 163,2 & 163,6 \\
\hline $\mathrm{NH}_{3}$ & 202,5 & 203,4 & 203,0 & 203,1 & 203,1 & 202,8 & 203,1 \\
\hline $\mathrm{C}_{2} \mathrm{H}_{2}$ & 152,3 & 152,9 & 152,9 & 152,9 & 153,0 & 152,5 & 152 \\
\hline $\mathrm{H}_{2}$ & 100,8 & 99,7 & 99,8 & 99,9 & 99,9 & 99,6 & 99,6 \\
\hline $\mathrm{HCl}$ & 133,6 & 133,7 & 132,7 & 132,3 & 132,4 & 133,1 & 133,1 \\
\hline $\mathrm{PH}_{3}$ & 187,1 & 186,7 & 186,1 & 186,1 & 186,1 & 186 & 187,6 \\
\hline $\mathrm{H}_{2} \mathrm{~S}$ & 168,8 & 168,7 & 167,1 & 167,4 & 167,5 & 167,9 & 167,7 \\
\hline $\mathrm{SiH}_{4}$ & 154,0 & 152,8 & 152,5 & 152,5 & 152,6 & 153,1 & 153,3 \\
\hline $\mathrm{Br}^{-}$ & 322,6 & 323,8 & 322,3 & 321,7 & 321,6 & 322,7 & 324,7 \\
\hline $\mathrm{CH}_{3} \mathrm{Br}$ & 157,3 & 157,9 & 156,9 & 156,5 & 156,5 & 157,5 & 159 \\
\hline \multirow{2}{*}{\multicolumn{2}{|c|}{$\begin{array}{l}\text { Desv. } \\
\text { Std. }\end{array}$}} & $\mathbf{0 , 7 8}$ & 0,65 & 0,56 & $\mathbf{0 , 5 8}$ & $\mathbf{0 , 6 3}$ & 1,01 \\
\hline & & 0,96 & $\mathbf{0 , 8 1}$ & $\mathbf{0 , 7 8}$ & $\mathbf{0 , 8 0}$ & $\mathbf{0 , 7 5}$ & 1,23 \\
\hline
\end{tabular}

Vale a pena ressaltar, informações relacionadas ao erro dos dados experimentais de referência, bem como essas medidas foram obtidas. Por exemplo, A.P. $\left(\mathrm{H}_{2} \mathrm{~S}, 298 \mathrm{~K}\right)$ $=168,8 \pm 1,27 \mathrm{kcal} \mathrm{mol}^{-1}$ [9] é obtido de uma média de dois resultados estimados para $\Delta_{\mathrm{f}} \mathrm{H}^{\circ}\left(\mathrm{H}_{3} \mathrm{~S}^{+}, 298 \mathrm{~K}\right)$. Por causa do alto valor na incerteza da energia de ligação do dímero de van der Waals o valor de referência no caso da A.P. $\left(\mathrm{H}_{2} \mathrm{O}, 298 \mathrm{~K}\right)=165,1 \pm$ $0,7 \mathrm{kcal}^{\mathrm{mol}}{ }^{-1}$ [9] é baseado na estimativa de cálculos teóricos e também em uma medição de equilíbrio de transferência de prótons. Dentre outros exemplos, pode-se sugerir que os cálculos aqui realizados podem ser tomados como informações confiáveis de elevada precisão na estimativa da A.P.

A inclusão de funções difusas tiveram um significado relevante sobre o cálculo de A.P. do Br-, provavelmente devido ao par de elétrons livre, sobretudo nos dois menores conjuntos de base avaliados nesse trabalho. O Desv. diminuiu para 0,70 e 0,60 kcal mol ${ }^{-1}$, com aug-cc-pVDZ e aug-cc-pVTZ, respectivamente.

\section{Conclusões}

A substituição da base 6-31G(d) pelas de correlação consistente, no método composto G3(MP2)//B3, continua a ser eficiente para cálculos moleculares de A.P. O menor desvio alcançado foi de apenas $0,56 \mathrm{kcal} \mathrm{mol}^{-1}$, com cc-pVQZ. Esse valor supera até mesmo métodos mais sofisticados como a teoria $\mathrm{G} 4$, que apresenta um desvio de $0,84 \mathrm{kcal} \mathrm{mol}^{-1}$ para o mesmo conjunto teste $\mathrm{G} 3 / 05$.

\section{Referências}

[1] Curtiss, LA; Redfern, PC; Raghavachari, K. WIREs Comput Mol Sci 2011, 1, 810.

[2] Morgon, NH; Coutinho, K. Métodos de química teórica e modelagem molecular. $1^{\mathrm{a}}$ ed. São Paulo: Editora Livraria da Física, 2007.

[3] Silva, GB; Leal, RC. Anais XIX Simp. Brasileiro de Química Teórica, 2017.

[4] Baboul, AG; Curtiss, LA; Redfern, PC; et al. J. Chem. Phys. 1999, 110, 7650.

[5] Rocha, CMR; Pereira, DH; Morgon, NH; Custódio, R. J. Chem. Phys. 2013, 139, 184108.

[6] Lima, JCB; Morgon, NH. Quím. Nova 2010, 33, 195.

[7] Heerdt, G; Morgon, NH. Quím. Nova 2011, 34, 868.

[8] Curtiss, LA; Redfern, PC; Raghavachari, K. J. Chem. Phys. 2005, 123, 124107.

[9] Hunter, EPL; Lias, SG. J. Phys. Chem. Ref. Data 1998, 27, 413. 